\title{
VALOR PERCEBIDO DE UM SISTEMA PRODUTO-SERVIÇO SUSTENTÁ VEL NUMA EMPRESA DE BIKE SHARING
}

Rolf Wolffenbüttel Neto (rolfneto@gmail.com) - Engenharia de Produção, Universidade Federal do Rio Grande do (UFRGS).

Maria Auxiliadora Cannarozzo Tinoco ( $\underline{\text { maria@ } @ \text { producao.ufrgs.br) }}$ - Departamento de Engenharia de Produção e Transportes, Universidade Federal do Rio Grande do (UFRGS).

Carla Schwengber ten Caten (tencaten@producao.ufrgs.br) - Escola de Engenharia, Universidade Federal do Rio Grande do (UFRGS).

Aline Marian Callegaro (aline.callegaro@ufrgs.br) - Departamento Interdisciplinar do Campus Litoral Norte, Universidade Federal do Rio Grande do (UFRGS).

\section{RESUMO}

Em busca de uma mobilidade urbana sustentável, nos últimos anos se destacou a proeminência dos sistemas de compartilhamento, os quais se baseiam no conceito de Sistema Produto-Serviço (ou Product-Service System - PSS). A literatura apresenta uma lacuna no que tange a percepção de valor dos clientes em ofertas PSS. Através do entendimento do valor percebido, é possível redesenhar os processos de uma oferta PSS que atenderá melhor às necessidades do cliente, a fim de atingir maior fidelização e vantagem competitiva sobre os concorrentes. Neste artigo é apresentado um instrumento para avaliar a percepção de valor dos clientes em relação à experiência de pedalar na cidade, visando identificar os atributos de valor prioritários de uma empresa de bike sharing. A partir das análises, foi possível realizar sugestões de melhorias para a entrega, as quais contemplem os principais atributos de valor priorizados pelos usuários.

Palavras chave: sistema produto-serviço, PSS, percepção de valor, compartilhamento de bicicletas, bike sharing 


\section{INTRODUÇÃO}

Cerca de 54\% da população mundial vive em áreas urbanas e se projeta que essa parcela aumentará para 70\% em 2050 (ONU, 2013). A crescente concentração de pessoas aumenta a demanda pelos diversos meios de locomoção. Para manter a prosperidade econômica e desenvolver uma melhor qualidade de vida, é necessário tornar sustentável a movimentação das pessoas nas cidades. Lam e Head (2012) definem mobilidade urbana sustentável como a facilidade, a conveniência, a disposição financeira e a acessibilidade para se locomover com o mínimo impacto ao meio ambiente.

Para atingir a mobilidade urbana sustentável, destaca-se o surgimento de modelos de negócios baseados no conceito de Product-Service Systems (PSS) ou sistemas produto-serviço (SOUSA-ZOMER et al., 2016). O PSS é uma combinação de produtos e serviços que, de forma sistêmica, entregam uma função ou uma utilidade desejada pelo cliente. A ênfase da proposta de valor está na venda do uso e da performance de um produto ao invés da posse do mesmo (BAINES et al., 2007). Os benefícios em termos de sustentabilidade provêm da quebra do conceito de lucro com volume de produção, reduzindo o consumo de recursos, aumentando a responsabilidade do fabricante, melhorando a eficiência no uso, a longevidade e a durabilidade do produto, além de ampliar a quantidade de material reutilizado (BOCKEN et al., 2014).

A mobilidade compartilhada já corresponde a $10 \%$ de todo transporte realizado na América do Norte, Europa e Ásia (ROLAND BERGER, 2014). Em cidades onde a cultura de compartilhamento está devidamente integrada com a infraestrutura de transporte, existe uma redução na posse de veículos privados e uma postergação na busca pela carteira de motorista pelos jovens (INSEE, 2015).

A popularização dos sistemas de compartilhamento (FISHMAN et al., 2014) somada ao potencial sustentável do PSS para a mobilidade urbana, é interessante para a literatura a realização de estudos empíricos que investiguem a operação do modelo de negócio desses sistemas. Uma lacuna da literatura está relacionada à percepção de valor do cliente em relação às ofertas de PSS. Autores sugerem que estudos sobre PSS sustentável explorem a demonstração e a avaliação de valor percebido dos clientes das novas ofertas de PSS sustentável em relação às tradicionais (PATALA et al., 2013). Ao compreender a percepção de valor dos clientes, é possível redesenhar o processo e conceito da oferta PSS que atenderá melhor às necessidades do cliente, a fim de atingir uma maior fidelização e uma vantagem competitiva sobre os concorrentes. 
Neste contexto, o presente artigo visa avaliar a percepção de valor dos clientes em relação à oferta PSS sustentável de uma startup de bike sharing em operação na cidade de Porto Alegre, a partir dos atributos de maior valor percebido para os clientes, no intuito de propor melhorias na oferta de PSS.

Na próxima seção desse artigo é apresentada uma breve revisão teórica sobre PSS, bike sharing e percepção de valor do cliente. Na seção seguinte é apresentada a metodologia do estudo, os resultados e discussões, por fim as conclusões.

\section{REVISÃO TEÓRICA}

A definição de PSS é trazida como um sistema vendável de produtos e serviços capazes de satisfazer as necessidades de um cliente (BOEHM e THOMAS, 2013), enfatizando a desmaterialização de produtos (TUKKER, 2015). Vasantha et al. (2012) ressalta que ainda não há consenso na literatura sobre a melhor abordagem, etapas e ferramentas para o desenvolvimento de ofertas PSS. Geum e ParK (2011) citam, por sua vez, a Product Service Blueprint para o mapeamento de processos, que possibilita a identificação das atividades-chave de maior valor para o cliente numa oferta de PSS.

No contexto da mobilidade urbana, os PSS aparecem com frequência sob o nome de mobilidade compartilhada (shared mobility), segmento de rápido crescimento da emergente sharing economy ou economia compartilhada (JIN et al., 2018), nos diferentes tipos de compartilhamento: carros (carsharing: ex. Car2Go); bicicletas (ridesharing: ex. Vélib); motocicletas; passagens; e caronas (ex. BlablaCar). Junto a estes, surge um a venda de pacotes de serviços de mobilidade (MaaS - Mobility as a Service: ex. ITS Finland) numa plataforma única (SPREI, 2017).

Os programas de bike sharing destacam-se por sua popularização (FISHMAN et al., 2014) e apresentam como principais desafios a necessidade de oferecer equipamentos de segurança, topografia e clima, os fatores culturais que tornam as pessoas dependentes dos carros, o roubo e o vandalismo (ONU, 2011). Por isso é importante identificar os principais fatores que levam as pessoas a usar ou não sistemas de bike sharing, uma vez que existem potenciais benefícios e sacrifícios envolvidos no seu uso. Como a percepção de valor do cliente pode ser definida como uma relação de troca entre os benefícios recebidos dos fornecedores e os custos sacrificados pelos consumidores (YANG e PETERSON, 2004; ZEITHAML, 1988), a sua avaliação pode ser de grande utilidade para os gestores de sistemas de bike sharing. Além 
disso, a aceitação e a satisfação do cliente dependem fortemente da sua percepção de valor frente à oferta, e a falta de entendimento da mesma é uma barreira para a adoção de um PSS (VEZZOLI et al., 2015).

O método proposto por Sheth et al. (1991) é uma das principais referências na modelagem de valor percebido por meio de cinco dimensões: funcional, social, emocional, epistêmica e condicional. São versões modificadas desse instrumento: PERVAL (SWEENEY e SOUTAR, 2001), SERV-PERVAL (PETRICK, 2002) e GLOVAL (SANCHEZ et al., 2006). O modelo TAM (technology acceptance model) adaptado foi utilizado para mensurar o valor percebido em sistemas de bike sharing (HAZEN et al., 2015; CHEN, 2016).

Na Figura 1 é apresentada a comparação das dimensões do valor percebido (modelo TAM, qualidade do serviço e da aceitação dos clientes) à escala PERVAL em sistemas de bike sharing. Observa-se que ainda não há definição da melhor abordagem e dos melhores métodos para tal, indicando a necessidade de novas ferramentas que capturem a percepção de valor a fim de avaliar a performance de um PSS.

\begin{tabular}{|c|c|c|}
\hline Autor & Dimen são u sada pelo autor pesquisa do & Dimensão na escala PERVAL \\
\hline \multirow{6}{*}{ Chen (2016) } & Prazer percebido & \multirow{2}{*}{ Emocional } \\
\hline & Atitude do cliente & \\
\hline & Utilidade percebida & \\
\hline & Facilidade de uso percebida & Funcional \\
\hline & Habilidade adquirida & \\
\hline & Normas subjetivas & Social \\
\hline \multirow{2}{*}{$\begin{array}{c}\text { Hazen et al. } \\
\text { (2015) }\end{array}$} & Qualidade percebida & Funcional \\
\hline & Conveniência percebida & Funcional/Emocional \\
\hline \multirow{3}{*}{$\begin{array}{l}\text { Kaplan et al. } \\
\text { (2015) }\end{array}$} & \multirow{3}{*}{$\begin{array}{c}\text { Teoria do Comportamento Planejado (TPB - } \\
\text { Theory of Planned Behaviour) }\end{array}$} & Emocional \\
\hline & & Funcional \\
\hline & & \\
\hline \multirow{2}{*}{ Morton (2018) } & Qualidade do Serviço & Funcional \\
\hline & Atitudes pró-ciclismo & Emocional/Social \\
\hline Nikitas (2018) & Atitudes pró-ciclismo & Emocional/Funcional/Social \\
\hline
\end{tabular}

FIGURA 1 - Analogia das dimensões utilizadas pelos diferentes modelos para mensurar valor percebido em bike sharing. Fonte: Autor. 


\section{METODOLOGIA}

Este trabalho foi desenvolvido na empresa ABC Bike Sharing, que gerencia um PSS de compartilhamento de bicicletas na cidade de Porto Alegre e acredita numa transformação cultural gradual, provendo uma oferta de mobilidade de qualidade, fidelizando os usuários ao uso da bicicleta no dia-a-dia. A ABC é uma empresa jovem que começou a operar em março de 2018, ao receber uma rodada de investimentos.

De acordo com a classificação de Tukker (2004), a ABC caracteriza-se como um PSS orientado ao uso. O sistema de compartilhamento de bicicletas da $\mathrm{ABC}$ é o chamado dockless ou free-floating, o qual permite que as bicicletas sejam estacionadas em qualquer lugar ao invés de estações fixas. A ABC busca inovar e apostar em um sistema híbrido para permitir que os usuários possam acessar e devolver as bicicletas em qualquer estabelecimento credenciado, como lojas, campi de universidades, prédios de escritórios, entre outros. Dessa forma, reduz os riscos de segurança do modelo free-floating, e permite maior flexibilidade, escalabilidade e menor burocracia para a criação de novas estações, uma vez que é mais simples concretizar uma parceria com um estabelecimento.

Para tornar esse modelo viável, a ABC conta com a tecnologia de cadeados smart (na roda traseira), com comunicação bluetooth para liberação por meio do aplicativo de celular da $\mathrm{ABC}$; e com os $\mathrm{ABCpoints,} \mathrm{que} \mathrm{são} \mathrm{estabelecimentos} \mathrm{credenciados,} \mathrm{os} \mathrm{quais} \mathrm{proveem} \mathrm{a}$ infraestrutura necessária para estacionar as bicicletas, por exemplo, a instalação do paraciclo. O estabelecimento usufrui de maior visibilidade, fluxo de pessoas (clientes da $\mathrm{ABC}$ ) e associação com uma marca sustentável. Junto a isso, a ABC vende o espaço de divulgação existente no paraciclo para que outras empresas façam propaganda de suas marcas, permitindo que elas recebam os dados de fluxo no ponto.

Esta pesquisa é de natureza aplicada; a abordagem é essencialmente qualitativa, apesar de envolver métodos quantitativos para mensurar atributos qualitativos; no que tange aos objetivos, é exploratória e descritiva pela elaboração do próprio procedimento de coleta de dados, visando interpretar as relações entre as variáveis que compõem o valor percebido; por fim, trata-se de uma pesquisa-ação devido à participação planejada e sistemática do pesquisador de forma cooperativa com a empresa em estudo (GERHARDT; SILVEIRA, 2009).

A metodologia foi dividida em sete etapas, sendo elas: i) desenho da oferta atual a partir da ferramenta Product Service Blueprint; ii) levantamento de atributos para mensurar o valor 
percebido; iii) construção do instrumento para avaliar o valor percebido; iv) aplicação do instrumento; v) análise dos resultados; vi) priorização dos atributos; vii) sugestões de melhorias.

A primeira etapa consistiu na utilização da ferramenta para design de PSS (GEUM e PARK, 2011) para visualizar o PSS de forma integral: (i) o uso do produto ao longo do seu ciclo de vida, (ii) o fluxo do serviço desde a gestão da empresa até o cliente, e (iii) o relacionamento das atividades de suporte integrando os componentes tangíveis (produto) e intangíveis (serviço). Observe a Figura 2.

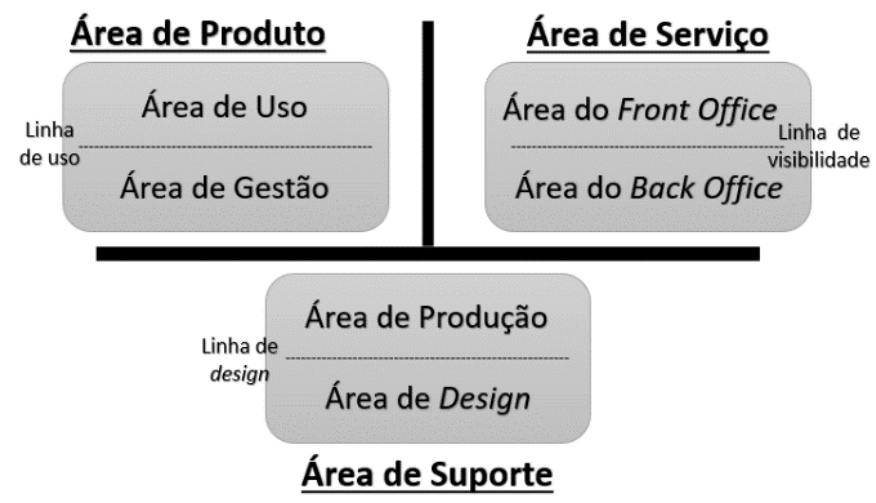

FIGURA 2 - Estrutura do Product Service Blueprint adaptado de Geum e Park (2011)

Na segunda etapa, foi realizada uma busca nos principais repositórios de publicações científicas de artigos que abordassem a avaliação de valor percebido em sistemas de bike sharing; utilizando as seguintes strings de pesquisa e suas combinações: value, bike sharing, perceived value, product service system, urban mobility, user perception. Foram selecionadas publicações a partir dos estudos de Chen (2016), Hazen et al. (2015), Kaplan et al. (2015) e Nikitas (2018) e consultados os sócios da $\mathrm{ABC}$.

$\mathrm{Na}$ terceira etapa, os atributos identificados foram agrupados em três dimensões, conforme semelhança: social, emocional, funcional (SWEENEY; SOUTAR; 2001). Devido ao apelo ambiental da empresa e das ofertas baseadas no conceito PSS sustentável, foi adicionada a quarta dimensão (ambiental). O instrumento para a avaliação de valor percebido foi construído na forma de um questionário composto de frases relacionadas a cada atributo na sua devida dimensão. Os itens poderiam ser pertinentes tanto a um benefício como a um sacrifício, conforme definição de valor percebido de Zeithaml (1988). Para avaliar o grau de concordância com cada atributo, foi selecionada a escala Likert de 1 a 5. Para avaliar o sacrifício monetário 
foi perguntando ao cliente o quanto ele estava disposto a pagar pelo uso da bicicleta (mínimo e máximo).

A quarta etapa consistiu na aplicação do instrumento. O questionário foi disponibilizado na internet e divulgado na base de usuários e nas redes sociais da $\mathrm{ABC}$, a qual sorteou um mês de utilização grátis das bicicletas para quem o divulgasse aos contatos (clientes potenciais). Os ciclistas e não-ciclistas deveriam responder brevemente o motivo por pedalarem ou deixarem de pedalar. Para definir o número de questionários necessários, foi considerado um coeficiente de variação moderado, erro relativo admissível de $5 \%$ e nível de significância moderado $\left(z_{\alpha / 2}=\right.$ 1,960). Usando como variáveis de estratificação a idade (jovem e adulto), gênero (masculino e feminino), tipo de uso (bike própria ou compartilhada) e frequência de uso semanal da bicicleta (zero ou não ciclista, um a três ou lazer, mais de três ou mobilidade), foi determinado como o mínimo 93 questionários com pelo menos 4 respostas para cada estrato (TRIOLA, 1998). Para validar a confiabilidade do instrumento, utilizou-se o método do Alfa de Cronbach (CRONBACH, 1951).

$\mathrm{Na}$ quinta etapa foi realizada a análise descritiva dos resultados dos questionários, cálculo da média e do desvio padrão de cada atributo. Na sexta etapa foram priorizados os atributos de maior valor percebido junto à empresa e, na sétima, realizadas as sugestões de melhorias.

\section{RESULTADOS E DISCUSSÃO}

O mapeamento dos processos de produção e entrega da oferta PSS da empresa foi realizado junto dos sócios da $\mathrm{ABC}$ durante uma visita técnica de um dos autores na empresa, contando com uma simulação de uso in loco. Ver as Figuras 3 e 4. 


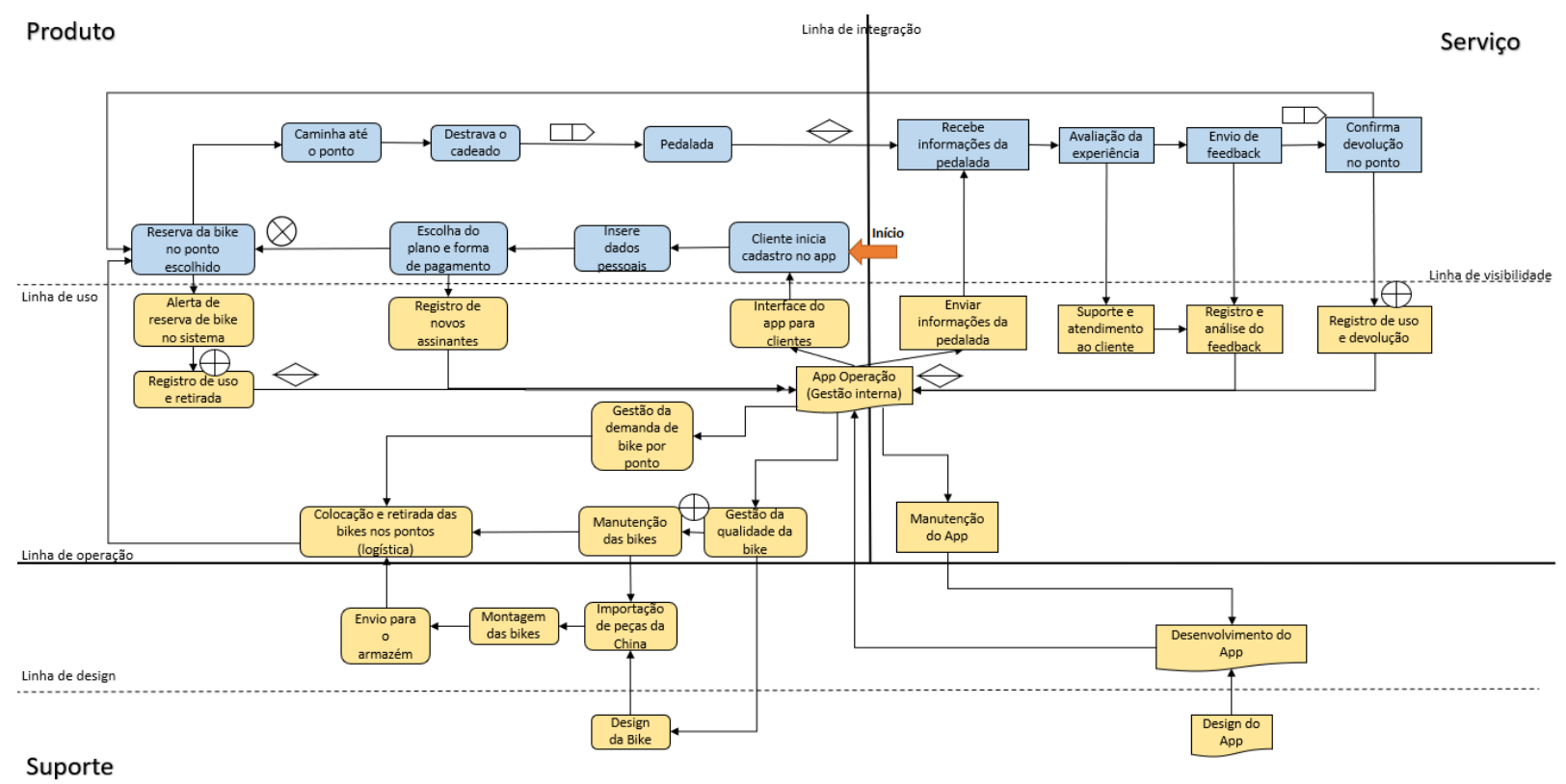

FIGURA 3 - PSS Blueprint inicial da empresa ABC. Fonte: Autor
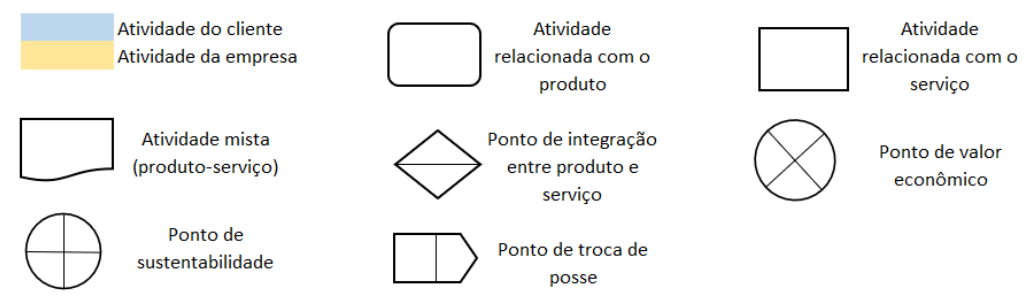

FIGURA 4 - Legenda do PSS Blueprint

O cliente inicia seu contato com a empresa ao realizar o cadastro no aplicativo, escolhe o plano e a forma de pagamento, reserva a bicicleta no ponto de retirada, desloca-se até o mesmo e destrava o cadeado. Após utilizar a bicicleta, o cliente recebe informações da pedalada e pode enviar um feedback para a empresa. Por fim, a bicicleta é devolvida e novamente travada num ponto. A empresa conta com um aplicativo interno para a gestão da demanda, da qualidade e da manutenção das bicicletas; e o suporte e atendimento ao cliente.

A ferramenta PSS Blueprint permitiu também a identificação do ponto do valor econômico - realização do pagamento e início da utilização do serviço; de troca de posse retirada e devolução da bicicleta; de integração - pedalada e compilação dos dados de uso para a disponibilização das bicicletas nos pontos; e de sustentabilidade - identificação do registro de uso da bicicleta, gestão da qualidade e manutenção. 


\subsection{Instrumento de percepção de valor construído}

O questionário construído foi composto por quatro dimensões de percepção de valor (cinco atributos cada) e teve como objetivo conhecer a opinião de ciclistas e não-ciclistas (aqueles que sabem andar de bicicleta, porém deixam de usá-la no dia-a-dia). Dessa forma, ao responder as perguntas iniciais de estratificação, o respondente era direcionado para o questionário mais adequado ao seu perfil, pois algumas questões foram modificadas para fazer sentido ao respondente.

O questionário contou também com duas perguntas referentes ao willing to pay, com a finalidade de entender o quão disposto o cliente está a pagar pelo serviço de bikesharing hoje, e o quanto ele pagaria caso todas necessidades dele fossem atendidas. Isso permite estimar o potencial de mercado que a $\mathrm{ABC}$ tem e o ganho monetário que eles podem ter, caso aperfeiçoem a entrega de valor.

\subsection{Resultados da aplicação do instrumento de percepção de valor}

Foram recebidas 186 respostas, com pelo menos 4 respostas por estrato. Foi obtido um escore de aproximadamente 0,85 no teste de confiabilidade de Alfa de Cronbach (software SPSS Statistics da IBM), validando o instrumento (CRONBACH, 1951).

Para validar a escolha das quatro dimensões de valor e demonstrar o potencial de crescimento do mercado de bikesharing em Porto Alegre, foi realizada uma nuvem de palavras a partir da frequência das respostas dos principais motivos para pedalar ou deixar de pedalar (Figura 5).

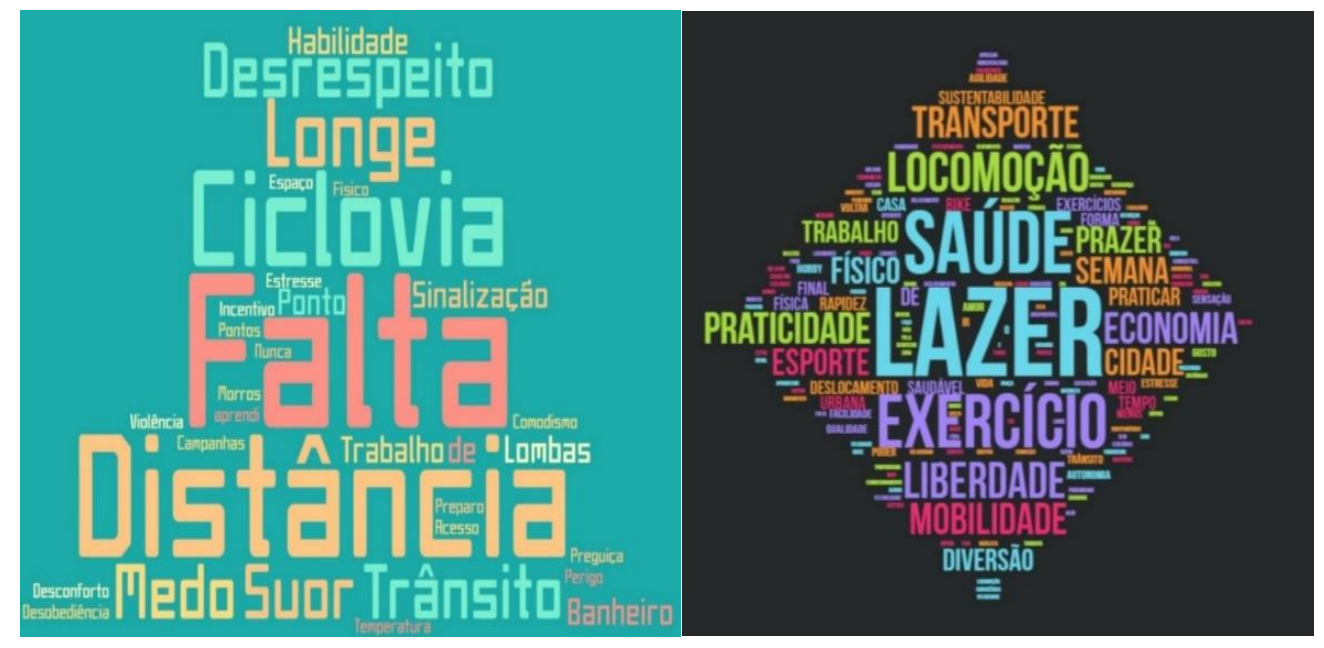

FIGURA 5 - Nuvem de palavras com os motivos para deixar de pedalar (à esquerda) e para pedalar (à direita) 
A nuvem de palavras mostra que os empecilhos para pedalar estão relacionados principalmente a fatores emocionais que derivam de aspectos funcionais, por exemplo, a sensação de medo e insegurança devido à falta de ciclovias. Nos motivos para pedalar, destacase novamente fatores emocionais e funcionais, como a preocupação com a saúde. Em segundo plano, aparece a palavra "sustentabilidade", relacionada com a dimensão ambiental, porém não há nada que exemplifique claramente os aspectos sociais.

A palavra "economia" destacada indica que a bicicleta possibilita redução de gastos na mobilidade do dia-a-dia. As respostas das willing to pay demonstram que os clientes estão dispostos a pagar entre 30 reais (preço atual praticado pela $A B C$ ) e 50 reais mensais por um serviço de bike sharing. Os adultos e não-ciclistas claramente estão dispostos a pagar mais pelo serviço; logo os clientes desse nicho representam um potencial de maior resultado monetário.

\subsection{Priorização dos atributos e melhorias sugeridas}

A priorização das dimensões de valor foi realizada a partir dos resultados das médias das notas dos atributos de cada dimensão, para os não-ciclistas e ciclistas, conforme demonstrado na Tabela 1.

TABELA 1 - Média das notas dos atributos de cada dimensão para os não-ciclistas e ciclistas

\begin{tabular}{lllll}
\hline Perfil & Emocional & Social & Funcional & Ambiental \\
\hline Não-ciclistas & 3,195 & 3,414 & 2,908 & 3,499 \\
Ciclistas & 4,376 & 3,642 & 3,873 & 4,145 \\
\hline
\end{tabular}

Dentre os ciclistas, os aspectos emocionais são os principais benefícios que geram valor percebido seguido do ambiental. Em comparação aos não-ciclistas, houve uma diferença na dimensão funcional, demonstrando que os ciclistas sentem que a bicicleta é um meio prático e flexível de mobilidade. É interessante notar que os não-ciclistas valorizam mais as dimensões ambiental e social.

A Tabela 2 demonstra os atributos priorizados no primeiro quartil para ciclistas e nãociclistas. 
TABELA 2 - Primeiro quartil de priorização dos atributos dos não-ciclistas e ciclistas

\begin{tabular}{|c|c|c|c|}
\hline Atributo & Perfil & Média & $\begin{array}{l}\text { Desvio } \\
\text { Padrão }\end{array}$ \\
\hline Não ter uma bicicleta própria é um obstáculo para eu pedalar. & Não-Ciclistas & 4,000 & 1,318 \\
\hline Eu me sentiria inseguro em andar no trânsito sem uma ciclovia. & Não-Ciclistas & 3,965 & 1,358 \\
\hline Eu percebo que as pessoas reconhecem positivamente quem anda de bike. & Não-Ciclistas & 3,847 & 1,118 \\
\hline Pedalar em um grupo de pessoas seria um ótimo programa de lazer. & Não-Ciclistas & 3,835 & 1,308 \\
\hline Eu me sentiria seguro pedalando com um amigo. & Não-Ciclistas & 3,624 & 1,123 \\
\hline Eu me sinto mais saudável andando de bike. & Ciclista & 4,812 & 0,542 \\
\hline Bike proporciona o sentimento de liberdade. & Ciclista & 4,693 & 0,628 \\
\hline Pedalar permite que eu viva mais a cidade e veja ela de outra forma. & Ciclista & 4,653 & 0,713 \\
\hline Andar de bike tira o estresse e me motiva no dia-a-dia. & Ciclista & 4,564 & 0,607 \\
\hline Para mim é essencial acessar uma bike de forma rápida [...] & Ciclista & 4,465 & 0,944 \\
\hline
\end{tabular}

Destaca-se para os não-ciclistas, o sentimento de insegurança frente à falta de habilidade e de ciclovias para retomar o hábito da bicicleta. Já para os ciclistas, foram priorizados os sentimentos de liberdade, alívio de estresse e de prazer. Esses sentimentos estão relacionados ao fato de que a maior parte dos clientes da empresa utilizarem a bicicleta como lazer. Para Chen (2016), o valor percebido tem efeitos positivos no prazer de usar bicicletas (satisfação), externalidades de rede e na fidelização de clientes.

Apesar da dimensão funcional ter tido menor relevância, o atributo de valor relacionado com o acesso à bicicleta aparece no primeiro quartil de priorização, sendo imprescindível para a experiência tanto de quem utiliza a bicicleta própria como a compartilhada. Achados semelhantes aos resultados de Hazen et al. (2015), os quais confirmam que o valor percebido em bikesharing é resultado da conveniência (facilidade de uso) e da qualidade (utilidade) percebida.

Para elaborar as melhorias, foi tomado como base a divisão dos atributos por quartil de priorização, as médias das dimensões, o willing to pay e a nuvem de palavras. Ver Figura 6. 


\section{Member-get-member}

Funcionalidade de bonificação por indicação de novos usuários que permaneçam ativos. A bonificação poderia ser estendida caso os dois usuários pedalem juntos com frequência no mês. A funcionalidade visa incluir, na base de clientes, os não-ciclistas que se sentiriam mais seguros pedalando com um amigo em momentos de lazer, especialmente os adultos que estão dispostos a pagar mais pelos serviços.

\section{Sugestão de trajeto}

Funcionalidade de sugestão de trajeto a ser percorrido, valorizando ruas com ciclovias, mais planas, com menos buracos e/ou mais amplas. A alimentação de dados seria a partir do próprio feedback do cliente sobre o trajeto percorrido.

\section{Ponto sob demanda}

Funcionalidade que permita ao clientes as sugestões de pontos de bicicleta ou a indicação de perímetro que melhor the atende para acessar e devolver a bicicleta.

\section{Clube de beneficios}

Criação de um sistema de troca de pontos obtidos nas pedaladas em benefícios (produtos, descontos, etc.) associados com marcas sustentáveis ou relacionadas com saúde e bem-estar. O sistema visa a formação de um clube de benefícios e de relações de parcerias com os Loopoints (essenciais no modelo de negócios da empresa).

FIGURA 6 - Sugestões de melhorias para os atributos priorizados.

\section{CONCLUSÃO}

O estudo teve como objetivo avaliar a percepção de valor de clientes reais e potenciais de uma empresa de bike sharing em relação à experiência de pedalar na cidade, visando identificar os atributos de valor prioritários e as sugestões de melhorias. Um instrumento foi construído e aplicado a clientes reais e potenciais da empresa em estudo.

As sugestões de melhorias realizadas estão direcionadas aos atributos de valor priorizados. As funcionalidades visam amenizar estresses emocionais durante a pedalada, facilitar o acesso aos pontos de bicicleta, reforçar a associação da marca da empresa à causa sustentável, e incluir a participação da base na captação de novos membros, tornando a comunidade mais ativa.

Para fins acadêmicos, o artigo contribui com a literatura ao construir um instrumento próprio para avaliar a percepção de valor de clientes em sistemas bike sharing, que pode ser replicado. Como estudos futuros, sugere-se investigar a influência dos diferentes perfis na percepção de valor; e a percepção de valor dos clientes dos aplicativos de bike sharing, essencial à entrega da oferta PSS. 


\section{REFERÊNCIAS}

BAINES, T. S. et al. State-of-the-art in product-service systems. Proceedings of the Institution of Mechanical Engineers, Part B: journal of engineering manufacture, v. 221, n. 10, p.1543-1552, 2007.

BOCKEN, N. M. P. et al. A literature and practice review to develop sustainable business model archetypes. Journal of cleaner production, v. 65, p. 42-56, 2014.

BOEHM, M.; THOMAS, O. Looking beyond the rim of one's teacup: a multidisciplinary literature review of Product-Service Systems in Information Systems, Business Management, and Engineering \& Design. Journal of Cleaner Production, v. 51, p. 245-260, 2013.

CHEN, S-Y. Using the sustainable modified TAM and TPB to analyze the effects of perceived green value on loyalty to a public bike system. Transportation Research Part A: Policy and Practice, v. 88, p. 58-72, 2016.

CRONBACH, L. J. Coefficient alpha and the internal structure of tests. Psychometrika, v. 16, n. 3, p. 297-334, 1951.

INSEE. En Ile-de-France: l'usage de la voiture pour aller travailler diminue, 2015. Disponível em: <https://www.insee.fr/fr/statistiques/1285604> Acesso em: 02 de abril de 2018.

FISHMAN, E.; WASHINGTON, S.; HAWORTH, N. Bike share's impact on car use: Evidence from the United States, Great Britain, and Australia. Transportation Research Part D: Transport and Environment, v. 31, p. 13-20, 2014.

GERHARDT, T. E.; SILVEIRA, D. T. Métodos de pesquisa. Plageder, 2009.

GEUM, Y.; PARK, Y. Designing the sustainable product-service integration: a product-service blueprint approach. Journal of Cleaner Production, v. 19, n. 14, p. 1601-1614, 2011.

HAZEN, B. T.; OVERSTREET, R. E.; WANG, Y. Predicting public bicycle adoption using the technology acceptance model. Sustainability, v. 7, n. 11, p. 14558-14573, 2015.

JIN, S. T. et al. Ridesourcing, the sharing economy, and the future of cities. Cities, 2018.

KAPLAN, S. et al. Intentions to use bike-sharing for holiday cycling: An application of the Theory of Planned Behavior. Tourism Management, v. 47, p. 34-46, 2015.

LAM, D.; HEAD, P. Sustainable urban mobility. In: Energy, Transport, \& the Environment. Springer, London, 2012. p. 359-371.

MORTON, C. Appraising the market for bicycle sharing schemes: Perceived service quality, satisfaction, and behavioural intention in London. Case Studies on Transport Policy, v. 6, p. 102-111, 2018.

NIKITAS, A. Understanding bike-sharing acceptability and expected usage patterns in the context of a small city novel to the concept: A story of 'Greek Drama'. Transportation Research Part F: Traffic Psychology and Behaviour, v. 56, p. 306-321, 2018.

ONU. State of the world's cities 2012/2013: Prosperity of cities. Routledge, 2013.

ONU. Bicycle-sharing schemes: enhancing sustainable mobility in urban areas. United Nations, Department of Economic and Social Affairs, p. 1-12, 2011.

PATALA, S. et al. A framework for developing sustainable value propositions for industrial product-service systems. In: IMP Conference, 29 ${ }^{\text {th }}, 2013$, Atlanta.

PETRICK, J. F. Development of a multi-dimensional scale for measuring the perceived value of a service. Journal of leisure research, v. 34, n. 2, p. 119-134, 2002.

SANCHEZ, J. et al. Perceived value of the purchase of a tourism product. Tourism management, v. 27, n. 3, p. 394-409, 2006.

ROLAND BERGER. Shared Mobility-How new businesses are rewriting the rules of the private transportation game. Roland Berger Strategy Consultants GmbH. München, 2014.

SHETH, J. N.; NEWMAN, B. I.; GROSS, B. L. Why we buy what we buy: A theory of consumption values. Journal of business research, v. 22, n. 2, p. 159-170, 1991. 
SOUSA-ZOMER, T.; CANTÚ, V. Z.; MIGUEL, P. A. C. Product-Service systems as sustainable alternatives to mobility: a comparative analysis of two bike-sharing systems. Brazilian Journal of Operations \& Production Management, v. 13, n. 3, p. 264-275, 2016.

SPREI, F. Disrupting mobility. Energy Research \& Social Science, 2017.

SWEENEY, J. C.; SOUTAR, G. N. Consumer perceived value: The development of a multiple item scale. Journal of retailing, v. 77, n. 2, p. 203-220, 2001.

TRIOLA, Mario F. Elementary Statistics. School Version. Addison-Wesley, 1998.

TUKKER, A. Eight types of product-service system: eight ways to sustainability? Experiences from SusProNet. Business strategy and the environment, v. 13, n. 4, p. 246-260, 2004.

TUKKER, A. Product services for a resource-efficient and circular economy-a review. Journal of cleaner production, v. 97, p. 76-91, 2015.

VAN HALEN, C.; VEZZOLI, C.; WIMMER, R. Methodology for product service system innovation: how to develop clean, clever and competitive strategies in companies. Uitgeverij Van Gorcum, 2005.

VASANTHA, G. V. A. et al. A review of product-service systems design methodologies. Journal of Engineering Design, v. 23, n. 9, p. 635-659, 2012.

VEZZOLI, Carlo et al. New design challenges to widely implement 'Sustainable Product-Service Systems'. Journal of Cleaner Production, v. 97, p. 1-12, 2015.

YANG, Z; PETERSON, R. T. Customer perceived value, satisfaction, and loyalty: The role of switching costs. Psychology \& Marketing, v. 21, n. 10, p. 799-822, 2004.

ZEITHAML, V. A. Consumer perceptions of price, quality, and value: a means-end model and synthesis of evidence. The Journal of marketing, p. 2-22, 1988. 